\title{
Inhibition of Osteoblast Proliferation and Migration by Exogenous and Endogenous Formaldehyde
}

Xu Teng ${ }^{b, c, *}$, Wei Huang ${ }^{b, c, *}$, Hefeng Yu ${ }^{b, c}$, Pei Wang ${ }^{b, c}$, Weishi Li ${ }^{a}$, Zhongqiang Chen ${ }^{a}$,

Dongwei Fan a, c,\#

${ }^{a}$ Department of Orthopaedics, Peking University Third Hospital, Beijing 100191, P.R. China

${ }^{b}$ Department of Biochemistry and Molecular Biology, Capital Medical University, Beijing

100069, P.R. China

${ }^{c}$ Beijing Key Laboratory for Cancer Invasion and Metastasis, Beijing 100069, P.R. China

*co-first authors

\#Address for correspondence

Dongwei Fan Ph.D. and M.D.

Department of Orthopaedics,

Peking University Third Hospital,

Beijing 100069, P.R. China

Phone: 86-10-82265557

Fax:86-10-82264217 


\begin{abstract}
Exogenous and endogenous formaldehyde (FA) plays an important role in cell growth and migration; however, its potential role in osteoblasts remains largely unclear. Cell counting kit-8 (CCK-8) and wound healing assays revealed that FA exposure at naturally occurring concentrations inhibited the proliferation and migration of mouse preosteoblast MC3T3E1 cells. Moreover, RNA sequencing (RNA-seq) analysis revealed that FoxO1 signaling pathway components displayed distinct expression patterns upon FA exposure, reflected through significant enrichment of cell migration. In particular, FoxO1、Sirt1 and FA-induced related protein expression which were closely with cell proliferation and migration were confirmed by western blotting. The present results indicate that the FoxO1 pathway is involved in FA-induced inhibition of cell growth and migration.
\end{abstract}

\title{
Keywords:
}

Formaldehyde, cell growth, cell migration, RNA-seq, FoxO1 


\section{Introduction}

Osteoblast growth and migration are essential for not only bone metabolism, including bone remodeling and responses to mechanical loading, but also bone pathological processes including bone repair after fractures and during osteoporosis [1-3].

Formaldehyde (FA) is one of the primary factors causing sick building syndrome. FA enters the body through inhalation from building materials, furniture, tobacco smoke, e-cigarettes, the sweetener aspartame, and, most directly, accidental consumption of methanol and insulation materials [4,5]. The WHO-recommended limit for indoor FA is 0.1 $\mathrm{mg} / \mathrm{m}^{3}$. However, indoor FA concentrations often exceed this recommended threshold. The average concentration of occupational FA exposure in Chinese factories is $1.37 \mathrm{mg} / \mathrm{m}^{3}$, which also exceeds the recommended threshold [6]. Simultaneously, FA is present in the natural environment, thus making it difficult to prevent FA exposure [7].

Furthermore, FA is produced endogenously, being found ubiquitously in cells owing to enzymatic oxidative demethylation by-products. Histones of the KDM1/JMJC or ABH enzyme family and the RNA and DNA demethylation machinery produce FA in the nucleus $[8,9]$. Moreover, FA can be endogenously produced by the action of neutrophil enzyme myeloperoxidase and $\mathrm{N}$-demethylation, a common biochemical 
phenomenon. Endogenous FA is present in the blood at 50-100 $\mu \mathrm{M}[10-$ $12]$.

Therefore, because of its abundance and chemical properties, formaldehyde can pose a significant risk to genomic integrity . However, limited information is available regarding the mechanisms underlying protection against FA at the cellular and organismal levels. It is unclear whether FA affects bone cells, especially osteoblasts and their proliferation and migration, which play essential roles in bone-associated diseases, e.g., bone repair during fracture and osteoporosis.

This study aimed to characterize the effect of formaldehyde on osteoblast growth and migration. First, we investigated the effect of FA on mouse preosteoblast MC3T3-E1 cells; thereafter, we attempted to determine the key signaling pathway affected in osteoblasts upon FA exposure via genome-wide transcriptional profiling analysis using RNAseq. 


\section{Materials and methods}

\subsection{Cell culture}

MC3T3-E1 cells were purchased from the ATCC (Manassas, VA, USA). Cells were cultured in a humidified atmosphere of $5 \% \mathrm{CO}_{2}$ and $95 \%$ air at $37^{\circ} \mathrm{C}$ in an alpha modification of Eagle's medium (Invitrogen Life Technologies, Carlsbad, CA, USA) supplemented with 10\% FBS, 100 $\mathrm{U} / \mathrm{mL}$ penicillin, and $0.1 \mathrm{mg} / \mathrm{mL}$ streptomycin[13].

\subsection{Cell proliferation}

Cell proliferation was assessed via a colorimetric tetrazolium saltbased assay, i.e., the cell counting-8 (CCK-8) assay. MC3T3-E1 cells were seeded at a density of 5000 cells/well into a 96-well plate and cultured in the absence or presence of FA at different concentrations $(100 \mu \mathrm{M}-500 \mu \mathrm{M})$. Thereafter, the CCK-8 solution (Dojindo) was added to each well, and the plate was incubated at $37^{\circ} \mathrm{C}$ in a $\mathrm{CO}_{2}$ incubator for 3 h. The absorbance was then determined at $450 \mathrm{~nm}$, using a microplate reader (Bio-Rad, Hercules, CA, USA).

\subsection{Wound-healing assay}

Cells were seeded in a 6-well plate the day before the assay, and cells were pre-treated with FA for $2 \mathrm{~h}, 6 \mathrm{~h}$ or $12 \mathrm{~h}$ before the wounds were inflicted on the cell monolayer. The wound was generated by scratching 
the cell monolayer with a pipette tip, and images were acquired at 0,12 , and $24 \mathrm{~h}$ after wounding. The degree of cell migration required to close the wound was analyzed using ImageJ software (National Institutes of Health, Bethesda, MD, USA).

\subsection{RNA preparation}

Total RNA was extracted using TRIzol reagent (Invitrogen, CA, USA, 15596026) on dry ice and processed in accordance with the manufacturer's instructions. To eliminate DNA, an aliquot of total RNA was treated with RQ1 RNase-Free DNase (Promega, WI, USA, M6101), followed by phenol/chloroform/isoamyl alcohol extraction and chloroform/isoamyl alcohol extraction, using Phase Lock Gel Light tubes (5 PRIME 2302800), followed by ethanol precipitation. Precipitated RNA was stored at $-20^{\circ} \mathrm{C}$ until use.

\subsection{Illumina RNA-Seq library construction}

Total RNA (20 mg) was used for poly(A)t selection using oligo(dT) magnetic beads (Invitrogen, CA, USA, 610-02), eluted in water, and used to generate an RNA-seq library, using the ScriptSeq kit (Epicentre, CA, USA SS10906). Libraries were amplified via polymerase chain reaction for $12-15$ cycles and sequenced in two lanes on the HiSeq 2000 platform at BGI Genome Center (Shenzhen, China).

2.6. RNA sequencing 
High-throughput sequencing was performed as paired-end 100 sequencing, using a HiSeq 2000 system (Illumina, San Diego, CA, USA). RNA-Seq reads were mapped using TopHat software (http://tophat.cbcb.umd.edu) to generate the alignment file, which was then used to assemble transcripts, estimate abundance, and detect differential expression of genes/isoforms, using cufflinks. Gene classification was based on searches in the DAVID (david.abcc.ncifcrf.gov) and MEDLINE (www.ncbi.nlm.nih.gov) databases.

\subsection{Illumina data analysis}

Raw reads were aligned to the B73 reference genome (RefGen v3), using TopHat 2.0.8 and STAR, with the minimum intron length set at 20 bp and the maximum intron length set at $50 \mathrm{~kb}$, with default settings for other parameters. Genes and isoforms were quantified using cufflinks version 2.2.1, using the GTF annotation file generated by PacBio sequencing. To reduce transcriptional noise, only isoforms/genes with FPKM values of $\geq 0.01$ (based on gene coverage saturation analysis) were included.

\subsection{In silico analysis}

To identify the pathways of intersecting genes, pathway enrichment analysis was performed using the Kyoto Encyclopedia of Genes and Genomes (KEGG, http://www.genome.ad.jp/kegg/). This analysis 
provides a better understanding of gene expression as a complete network. The Fisher's exact test and Chi-square test were performed, and the threshold for statistical significance was defined by a P-value of $<0.05$ and the false discovery rate. Furthermore, we performed gene-set enrichment analysis, using GSEA v2.2.2 software and a c5.all. v5.1. symbols.gmt (gene ontology)-gene set matrix.

\subsection{Western blot analysis}

MC3T3-E1 cells were cultured in a 10-cm culture dish at $5 \times 10^{5}$ cells $/ \mathrm{mL}$ at $37^{\circ} \mathrm{C}$ in $5 \% \mathrm{CO}_{2}$ in medium supplemented with $\mathrm{FA}$ at different concentrations. Thereafter, cells were harvested, and extracts were cleared via centrifugation (Thermo Scientific, CA, USA). Protein concentrations were determined using the BCA protein assay kit (Thermo Scientific, CA, USA). Protein samples (50 $\mu \mathrm{g}$ each) were separated via SDS-PAGE (12\% resolving gel) and then transferred to polyvinylidene difluoride membranes. The membranes were blocked with blocking solution containing 3\% BSA and $0.1 \%$ Tween-20 for $30 \mathrm{~min}$ and probed overnight at $4{ }^{\circ} \mathrm{C}$ with the following primary antibodies: anti-FoxO1, antiSIRT1, anti-PRMT6, anti-SUZ12, anti-EZH2 (1:500 each) (Merck Millipore, Darmstadt, Germany), and anti-GAPDH (1:5000) (Bioworld Technology, Saint Louis Park, MN, USA). The membrane was then probed with HRP-labeled goat anti-rabbit $\operatorname{IgG}(\mathrm{H}+\mathrm{L})$ secondary 
antibody (1:1000; KPL, Milford, MA,USA) for $1 \mathrm{~h}$. Finally, protein bands were detected using $\mathrm{DAB}$ reagent. Relative protein expression levels were determined using Quantity One (Bio-Rad).

\subsection{Statistical analysis}

Unless indicated otherwise, data are presented as mean values \pm standard deviation (SD) values and analyzed using one-way ANOVA, followed by the Dunnett multiple comparison test for post hoc analysis. A $p$ value $<0.05$ was considered statistically significant. Data were analyzed using SPSS11 (Chicago, IL, USA) statistical software.

\section{Results}

\subsection{Effect of FA on osteoblast proliferation}

The effect of FA on proliferation in MC3T3-E1 osteoblasts was determined using the CCK-8 assay. As shown in Fig. 1, cell proliferation was observed upon treatment with FA at different concentrations for $2 \mathrm{~h}$ (Fig. 1. upper panel) and $6 \mathrm{~h}$ (Fig. 1. lower panel). Typically, each group treated with $100 \mu \mathrm{M}, 150 \mu \mathrm{M}, 200 \mu \mathrm{M}, 300 \mu \mathrm{M}$, and $500 \mu \mathrm{M}$ FA markedly inhibited proliferation in comparison with untreated cells, and longer the duration of FA treatment, stronger the inhibition of cell proliferation. These results notably indicate the strong inhibitory effect of 
FA on MC3T3-E1 cells through reduced cell viability in a dosedependent manner within 0-96 h (Fig. 1).

\subsection{Effect of FA on osteoblast migration}

We investigated the effect of FA on the migration of MC3T3-E1 cells, using a wound-healing assay. Upon treatment of cells for $2 \mathrm{~h}, 6 \mathrm{~h}$ and 12 h with $100 \mu \mathrm{M}, 150 \mu \mathrm{M}, 200 \mu \mathrm{M}, 300 \mu \mathrm{M}$, and $500 \mu \mathrm{M}$ FA, respectively, wounds were inflicted on the surface of the confluent cellular monolayer surface, and cells were allowed to migrate for 12 and $24 \mathrm{~h}$. The wound healing assays showed that FA significantly decreased the rate of wound closure of MC3T3-E1 cells in comparison with the control at $12 \mathrm{~h}$ and 24 h of treatment with $200 \mu \mathrm{M}$ to $500 \mu \mathrm{M}$ FA ( $p<0.05$, Dunnett comparison test) (Fig. 2). In addition, whether the cells were treated for 2 h, 6 h, or 12 $\mathrm{h}$, the wound closure rate of MC3T3-E1 cells decreased with the increase of FA dose, showing a dose-dependent inhibition of cell migration.

3.3. Identification of the candidate signaling pathway involved in cell proliferation and migration after FA treatment

To explore the potential mechanism underlying osteoblast proliferation and migration after FA treatment at the whole-genome level, we performed a genome-wide transcriptional analysis via RNA-seq. For cells treated with each FA concentration, whole-genome transcriptomes were determined. In brief, after treatment of MC3T3-E1 cells with 100 
$\mu \mathrm{M}$ and $300 \mu \mathrm{M}$ FA for $6 \mathrm{~h}$, cells were harvested for RNA extraction and subjected to RNA-seq. Preliminary results indicate that $100 \mu \mathrm{M}$ and 300 $\mu \mathrm{M}$ FA differentially up- and downregulated numerous genes (Fig. 3A), and the differentially expressed genes were enriched upon KEGG pathway analysis, indicating that these genes are involved in cell adhesion. Signaling pathways such as those involving FoxO, Wnt, autophagy-related proteins, MAPK, mTOR, Hippo, and p53 (Fig. 3B) are involved in cell growth, proliferation, migration, and invasion. The 972 differentially upregulated genes and the 3968 differentially downregulated genes were separately enriched in the KEGG pathway. These upregulated genes were found to be primarily involved in DNA damage repair, cell metabolism, and other signaling pathways, while the downregulated genes were primarily involved in p53, Hippo, autophagy, FoxO, Wnt, TGF-beta, and other signaling pathways (Fig. 3C). Furthermore, analysis of the RNA-seq results via GSEA revealed that the FA-300 group significantly inhibited signaling pathways such as FoxO, p53, Wnt, and mTOR ( $p<0.05$, Dunnett multiple comparison test) (Fig. 3D). These results preliminarily indicate that the in vitro MC3T3-E1 osteoblast model of FA treatment is indeed reliable.

\subsection{Effect of FA on FOXO1/SIRT1 protein expression}

To evaluate effect of FA on FOXO1 [14], SIRT1 , PRMT6 , SUZ12 [15], and EZH2 [16] protein expression levels, which are closely 
associated with cell proliferation and migration in MC3T3-E1 cells, we treated cells with FA at increasing concentrations from $100 \mu \mathrm{M}$ to 300 $\mu \mathrm{M}$. Western blot analysis revealed that FOXO1, SIRT1, PRMT6, SUZ12, and EZH2 were significantly downregulated upon FA treatment (Fig. 4).

\section{DISCUSSION}

To our knowledge, this study is the first to report that FA, a product of cellular metabolism and a ubiquitous environmental toxin, suppressed osteoblastic cell proliferation and decreased cell migration at naturally occurring concentrations. Furthermore, a genome-wide transcriptional analysis via RNA-seq performed to explore the potential mechanism underlying FA stimulation revealed distinct expression patterns of components of the FoxO signaling pathway upon FA treatment, reflected through significantly decreased cell migration and proliferation.

Forkhead box O1 (FoxO1), also known as the forkhead rhabdomyosarcoma transcription factor (FKHR), belongs to the Forkhead box (FOX) family and is a key transcriptional regulator in cell proliferation, differentiation, and migration via a receptor tyrosine kinase signaling pathway $[17,18]$. Recent studies have reported that osteoblast growth and migration is regulated by FoxO factors $[19,20]$. FoxO1 is the primary regulator of redox balance and function in osteoblasts among 
three key members of the FoxO family, i.e., FoxO1, FoxO3a, and FoxO4. Depletion of FoxO1 in osteoblasts reportedly resulted in decreased proliferation and bone volume in $\mathrm{FoxO}_{\mathrm{Ob}^{-/-}}$mice $[21,22]$. In the present study, genome-wide expression profiling revealed that $F O X O 1$ was downregulated upon FA exposure. Furthermore, using protein semiquantification techniques, FoxO1 was downregulated at higher FA concentrations.

Transcription-related factors can be integrated into specific DNA sites, and this molecular process is regulated by the configuration changes of chromatin. However, highly condensed chromatin is relieved through chromatin remodeling of itself with the alteration of covalence power between histone and DNA chain, and this mechanism may control gene expression and silencing.

Recently, several studies demonstrated a critical role of the Prmt6, Suz12, Ezh2 that can not only affect cell proliferation and differentiation through chromatin remodeling, but this process is related to FoxO signaling pathway. For example, oxidative stress in cells, activation of SIRT/FOXO/SOD2 signaling pathway, in turn, recruits chromatin remodeling complexes (Prmt6, Suz12, Ezh2) to activate related gene expression[23]. Our genome-wide analysis found that not only the FOXO1 and SIRT1 expression was reduced, but also the expression of 
chromatin remodeling key proteins including PRMT6, SUZ12, and EZH2 was down-regulated, and further confirmed by Western Blot.

These data indicate that FoxO1 plays an important role in FA-induced inhibition of proliferation and migration in MC3T3-E1 cells, and further studies are intended to clarify its upstream and downstream interacting partners to further clarify the detailed mechanism of action of FA on cell proliferation and migration. In the present study, transcriptomic analysis of cellular responses elucidated the potential underlying mechanism and the dose-dependent response pattern.

Although the present results cannot be extrapolated directly to the in vivo setting, further studies are required to identify potentially sensitive target genes, since dysregulated expression of these genes can affect osteogenesis and cause bone disease.

In conclusion, the present results show that FA inhibits cell migration and proliferation in MC3T3-E1 cells. Furthermore, this newly identified effect of FA on cell migration can be harnessed not only to prevent air pollution but also for novel therapeutic applications such as acceleration of wound healing by accumulation of preosteoblast cells at the bone fracture site. Therefore, further studies are required to clarify the effect of FA on FoxO1 and other target molecules mediating FA-induced inhibition of cell migration and proliferation. 


\section{Conflicts of interest}

There is no conflict of interests in this work.

\section{Acknowledgements}

*This work was supported by grants from National Natural Science

Foundation of China (81672726; 30801156); National Key Research and

Development Program of China (2017YFC0113001); Program for

Training Capital Science and Technology Leading Talents

(Z181100006318003); and Fostering Young Scholars of Peking

University Health Science Center (BMU2017PY015) 


\section{Reference}

1. Thiel A, Reumann MK, Boskey A, Wischmann J, von Eisenhart-Rothe R, MayerKuckuk P Osteoblast migration in vertebrate bone. Biol Rev Camb Philos Soc. 2018; 93(1): 350-363.doi:10.1111/brv.12345 PMID 28631442.

2. Movilla N, Borau C, Valero C, Garcia-Aznar JM Degradation of extracellular matrix regulates osteoblast migration: A microfluidic-based study. Bone. 2018; 107(10-17.doi:10.1016/j.bone.2017.10.025 PMID 29107125.

3. Deb Roy A, Yin T, Choudhary S, Rodionov V, Pilbeam CC, Wu YI Optogenetic activation of Plexin-B1 reveals contact repulsion between osteoclasts and osteoblasts. Nat Commun. 2017; 8(15831.doi:10.1038/ncomms15831 PMID 28635959.

4. Jensen RP, Luo W, Pankow JF, Strongin RM, Peyton DH Hidden formaldehyde in ecigarette aerosols. N Engl J Med. 2015; 372(4): 392394.doi:10.1056/NEJMc1413069 PMID 25607446.

5. Pankow JF, Strongin RM, Peyton DH More on hidden formaldehyde in e-cigarette aerosols. N Engl J Med. 2015; 1577.doi:10.1056/NEJMc1502242 PMID 25875273.

6. Tang X, Bai Y, Duong A, Smith MT, Li L, Zhang L Formaldehyde in China: production, consumption, exposure levels, and health effects. Environ Int. 2009; 35(8): 1210-1224.doi:10.1016/j.envint.2009.06.002 PMID 19589601.

7. Bolt HM, Morfeld $P$ New results on formaldehyde: the 2nd International Formaldehyde Science Conference (Madrid, 19-20 April 2012). Arch Toxicol. 2013; 87(1): 217-222.doi:10.1007/s00204-012-0966-4 PMID 23138381.

8. Dreyfuss JH Occupational formaldehyde exposure linked to increased risk of myeloid leukemia and death. CA Cancer J Clin. 2010; 60(3): 135136.doi:10.3322/caac.20071 PMID 20375322.

9. Pontel LB, Rosado IV, Burgos-Barragan G, Garaycoechea JI, Yu R, Arends MJ, et al. Endogenous Formaldehyde Is a Hematopoietic Stem Cell Genotoxin and Metabolic Carcinogen. Mol Cell. 2015; 60(1): 177188.doi:10.1016/j.molcel.2015.08.020 PMID 26412304.

10. Tan SLW, Chadha S, Liu Y, Gabasova E, Perera D, Ahmed K, et al. A Class of Environmental and Endogenous Toxins Induces BRCA2 Haploinsufficiency and Genome Instability. Cell. 2017; 169(6): 1105-1118 e1115.doi:10.1016/j.cell.2017.05.010 PMID 28575672.

11. Luo W, Li H, Zhang Y, Ang CY Determination of formaldehyde in blood plasma by high-performance liquid chromatography with fluorescence detection. J Chromatogr B Biomed Sci Appl. 2001; 753(2): 253-257PMID 11334338.

12. Heck HD, Casanova-Schmitz M, Dodd PB, Schachter EN, Witek TJ, Tosun T Formaldehyde $(\mathrm{CH} 2 \mathrm{O})$ concentrations in the blood of humans and Fischer344 rats exposed to $\mathrm{CH} 2 \mathrm{O}$ under controlled conditions. Am Ind Hyg Assoc J. 1985; 46(1): 1-3.doi:10.1080/15298668591394275 PMID 4025145.

13. Luttrell LM, Dar MS, Gesty-Palmer D, El-Shewy HM, Robinson KM, Haycraft C], et al. Transcriptomic characterization of signaling pathways associated with osteoblastic differentiation of MC-3T3E1 cells. PLoS One. 2019; 14(1): e0204197.doi:10.1371/journal.pone.0204197 PMID 30608923.

14. Cabrera-Ortega AA, Feinberg D, Liang Y, Rossa C, Jr., Graves DT The Role of Forkhead Box 1 (FOXO1) in the Immune System: Dendritic Cells, T Cells, B Cells, and Hematopoietic Stem Cells. Crit Rev Immunol. 2017; 37(1): 113.doi:10.1615/CritRevImmunol.2017019636 PMID 29431075. 
15. Lee SR, Roh YG, Kim SK, Lee JS, Seol SY, Lee HH, et al. Activation of EZH2 and SUZ12 Regulated by E2F1 Predicts the Disease Progression and Aggressive Characteristics of Bladder Cancer. Clin Cancer Res. 2015; 21(23): 53915403.doi:10.1158/1078-0432.CCR-14-2680 PMID 26268246.

16. Shan L, Zhou X, Liu X, Wang Y, Su D, Hou Y, et al. FOXK2 Elicits Massive Transcription Repression and Suppresses the Hypoxic Response and Breast Cancer Carcinogenesis. Cancer Cell. 2016; 30(5): 708722.doi:10.1016/j.ccell.2016.09.010 PMID 27773593.

17. Riddell M, Nakayama A, Hikita T, Mirzapourshafiyi F, Kawamura T, Pasha A, et al. aPKC controls endothelial growth by modulating c-Myc via FoxO1 DNAbinding ability. Nat Commun. 2018; 9(1): 5357.doi:10.1038/s41467-01807739-0 PMID 30559384.

18. Siqueira MF, Flowers S, Bhattacharya R, Faibish D, Behl $Y$, Kotton DN, et al. FOXO1 modulates osteoblast differentiation. Bone. 2011; 48(5): 10431051.doi:10.1016/j.bone.2011.01.019 PMID 21281751.

19. Ferrando $M$, Wan $X$, Meiss $R$, Yang J, De Siervi A, Navone $N$, et al. Heme oxygenase-1 (HO-1) expression in prostate cancer cells modulates the oxidative response in bone cells. PLoS One. 2013; 8(11): e80315.doi:10.1371/journal.pone.0080315 PMID 24224047.

20. Almeida M, Han L, Ambrogini E, Weinstein RS, Manolagas SC Glucocorticoids and tumor necrosis factor alpha increase oxidative stress and suppress Wnt protein signaling in osteoblasts. J Biol Chem. 2011; 286(52): 4432644335.doi:10.1074/jbc.M111.283481 PMID 22030390.

21. Kim KM, Park SJ, Jung SH, Kim EJ, Jogeswar G, Ajita J, et al. miR-182 is a negative regulator of osteoblast proliferation, differentiation, and skeletogenesis through targeting FoxO1. J Bone Miner Res. 2012; 27(8): 1669-1679.doi:10.1002/jbmr.1604 PMID 22431396.

22. Rached MT, Kode A, Xu L, Yoshikawa Y, Paik JH, Depinho RA, et al. FoxO1 is a positive regulator of bone formation by favoring protein synthesis and resistance to oxidative stress in osteoblasts. Cell Metab. 2010; 11(2): 147160.doi:10.1016/j.cmet.2010.01.001 PMID 20142102.

23. Kenny TC, Hart P, Ragazzi M, Sersinghe M, Chipuk J, Sagar MAK, et al. Selected mitochondrial DNA landscapes activate the SIRT3 axis of the UPR $(\mathrm{mt})$ to promote metastasis. Oncogene. 2017; 36(31): 43934404.doi:10.1038/onc.2017.52 PMID 28368421. 


\section{Figure legends}

Fig. 1. Effects of formaldehyde (FA) on cell viability in MC3T3-E1.

MC3T3-E1 cells were treated for $2 \mathrm{~h}(\mathrm{~A})$ and $6 \mathrm{~h}(\mathrm{~B})$ with the control or with FA at different concentrations, and cell viability was examined using the cell counting kit- 8 assay at $0,24,48,72$, and $96 \mathrm{~h}$. The results are expressed as the mean $\pm \mathrm{SD}$ values of at least three independent experiments. ${ }^{*} p<0.05,{ }^{* *} p<0.01$.

Fig. 2. Effects of formaldehyde (FA) on cell migration in MC3T3-E1.

Cells were treated for $2 \mathrm{~h}(\mathrm{~A}), 6 \mathrm{~h}(\mathrm{C})$ and $12 \mathrm{~h}(\mathrm{E})$ with the control or with FA at different concentrations, and cell migration was examined via a wound-healing assay. Statistical analysis of migrations in each group with $12 \mathrm{~h}$ (left panel) and $24 \mathrm{~h}$ (right panel) were shown in (B), (D) and (F) panel. The results are expressed as mean \pm SD values of at least three independent experiments. ${ }^{*} p<0.05,{ }^{*} p<0.01$.

Fig. 3. MC3T3-E1 cells treated with $100 \mu \mathrm{M}$ and $300 \mu \mathrm{M}$ formaldehyde (FA) for RNA-seq and analysis of RNA-seq results.

(A) RNA-seq revealed that 972 genes were upregulated and 3968 genes were downregulated (fold-change $>2$ ) upon treatment with $100 \mu \mathrm{M}$ and $300 \mu \mathrm{M}$ FA. (B) Differential gene enrichment in KEGG pathways upon treatment with $100 \mu \mathrm{M}$ and $300 \mu \mathrm{M} \mathrm{FA}$, showing that these genes are 
involved in cell adhesion, Wnt, autophagy, MAPK, FoxO, mTOR, Hippo, and p53 signaling pathways. (C) The 972 differentially upregulated genes and the 3968 differentially downregulated genes were separately enriched in the KEGG pathway. (D) GSEA analysis of RNA-seq results.

Fig. 4. Altered FoxO1 expression levels in MC3T3-E1 cells after FA treatment.

MC3T3-E1 were seeded in 6-well plates and treated with formaldehyde (FA). FoxO1 expression levels and FA-induced related protein expression levels were determined via western blot analysis. 
A

MC3T3-E1 exposed to formaldehyde(FA) for $2 \mathrm{~h}$

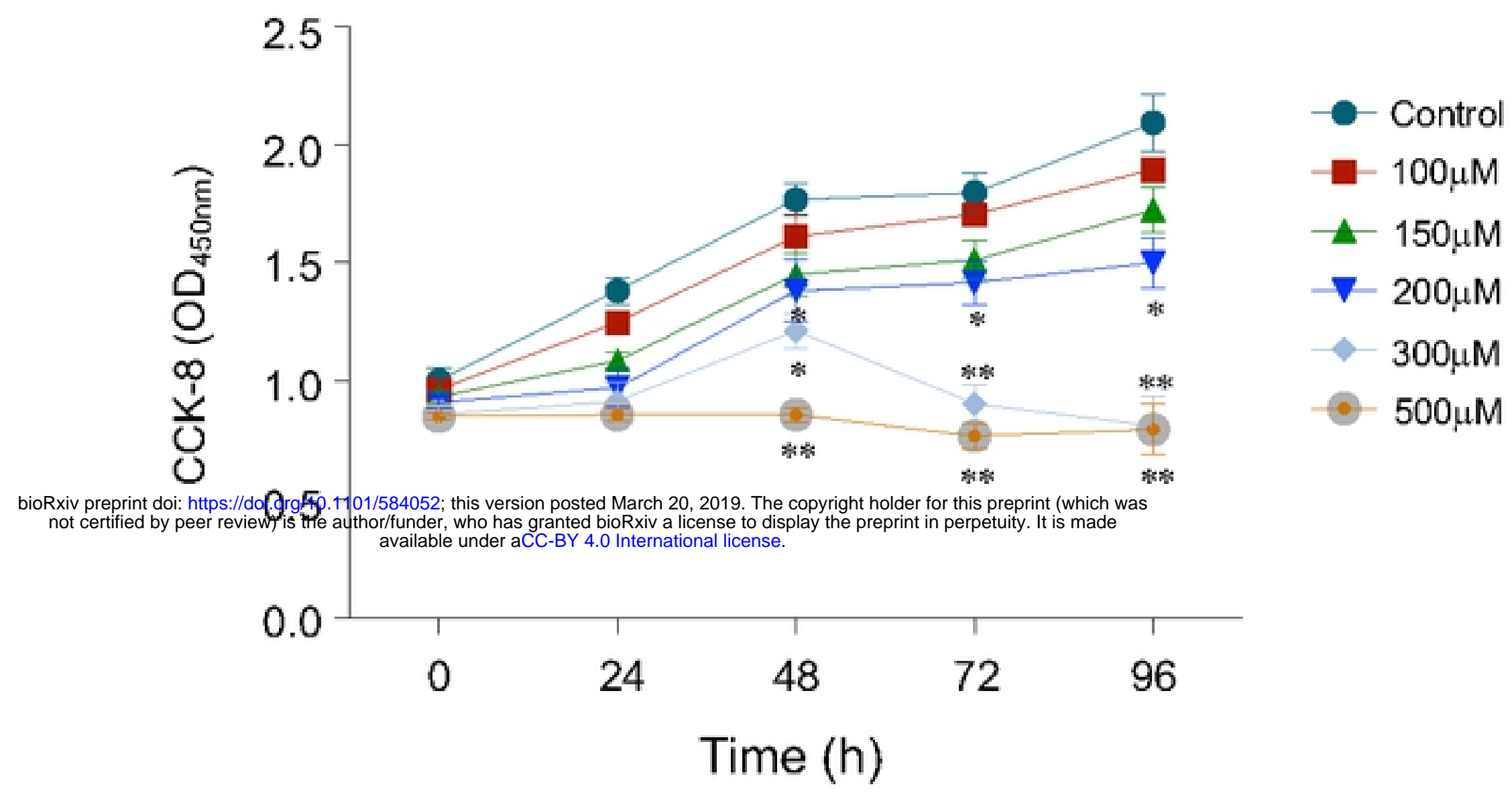

B

MC3T3-E1 exposed to formaldehyde(FA) for $6 \mathrm{~h}$

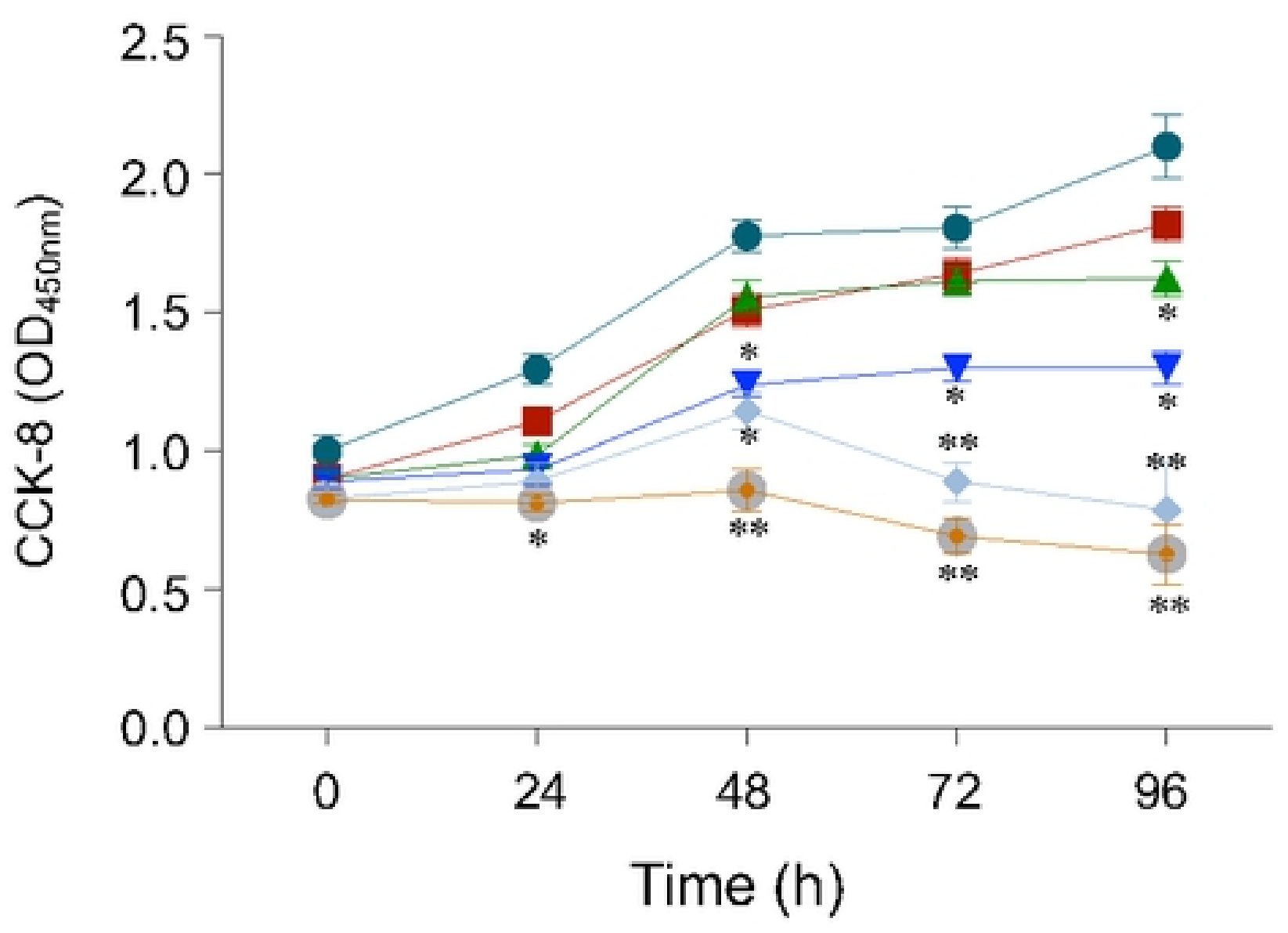

- Control
$-100 \mu \mathrm{M}$
$-150 \mu \mathrm{M}$
$\nabla-200 \mu \mathrm{M}$
$-300 \mu \mathrm{M}$
$-500 \mu \mathrm{M}$

Figure-1 


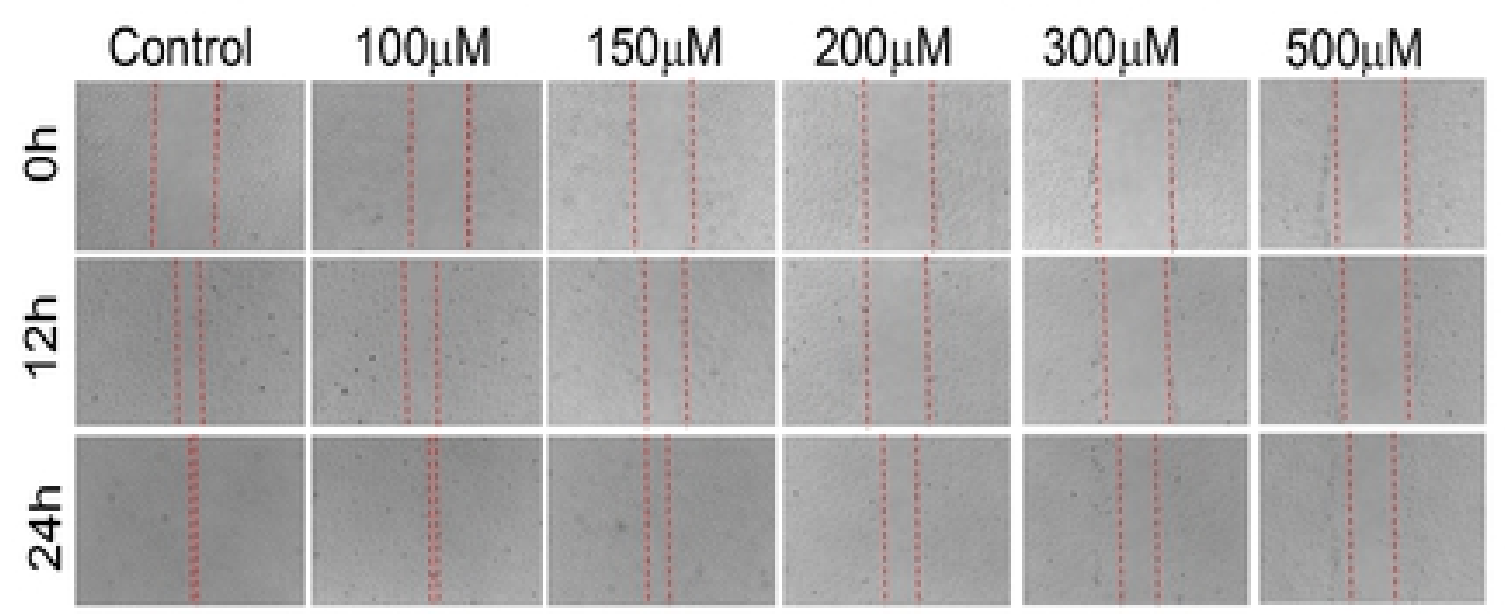

B

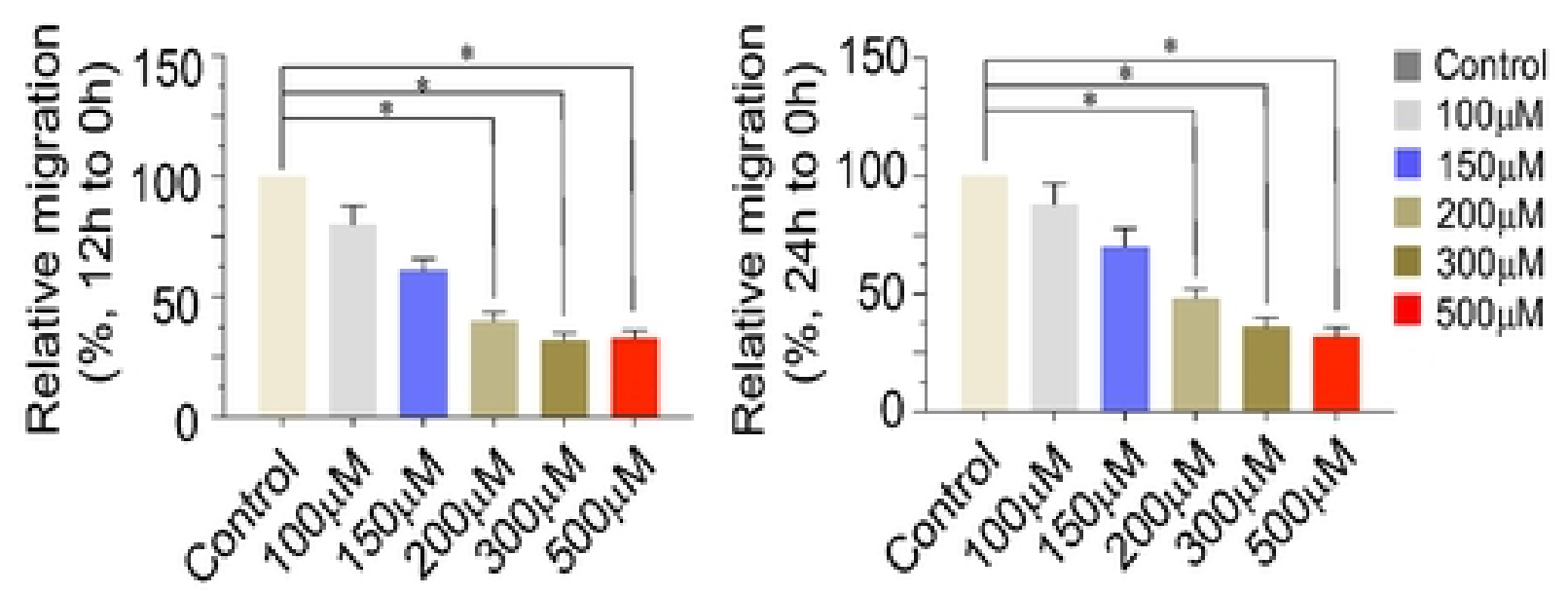

bioRxiv preprint doi: https://doi.org/10.1101/584052; this version posted March 20, 2019. The copyright holder for this preprint (which was

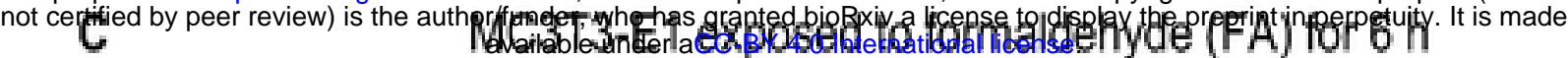

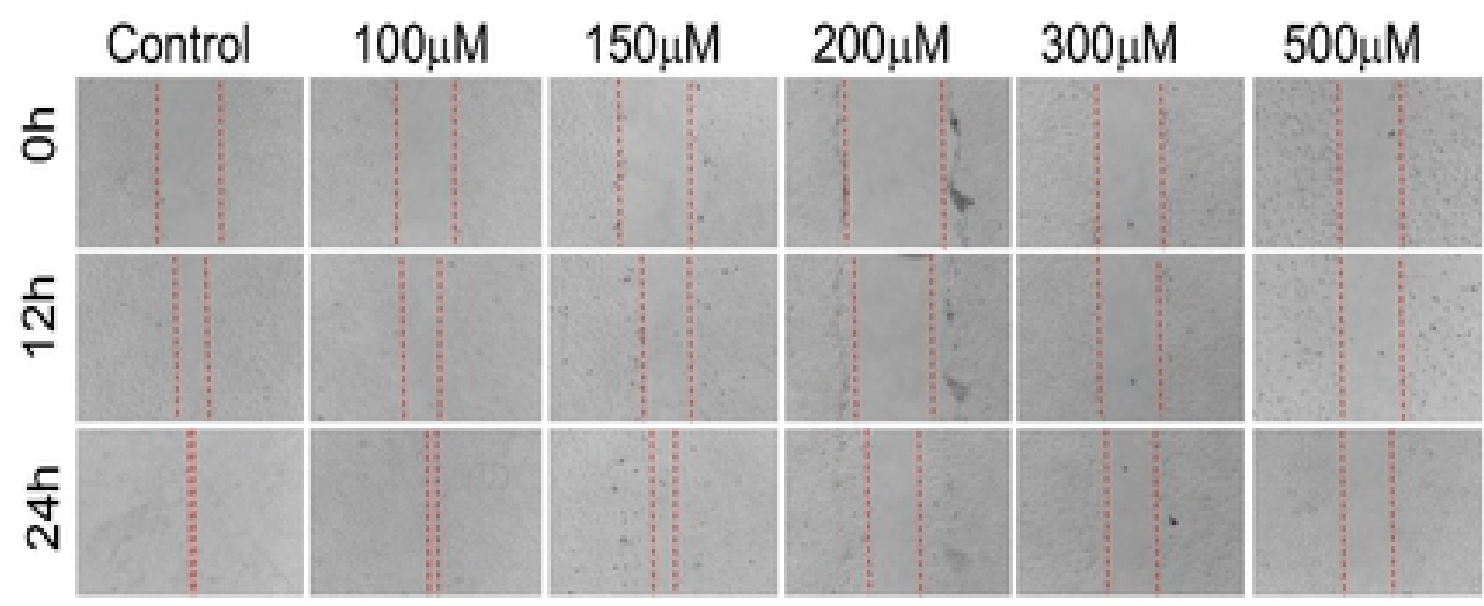

D

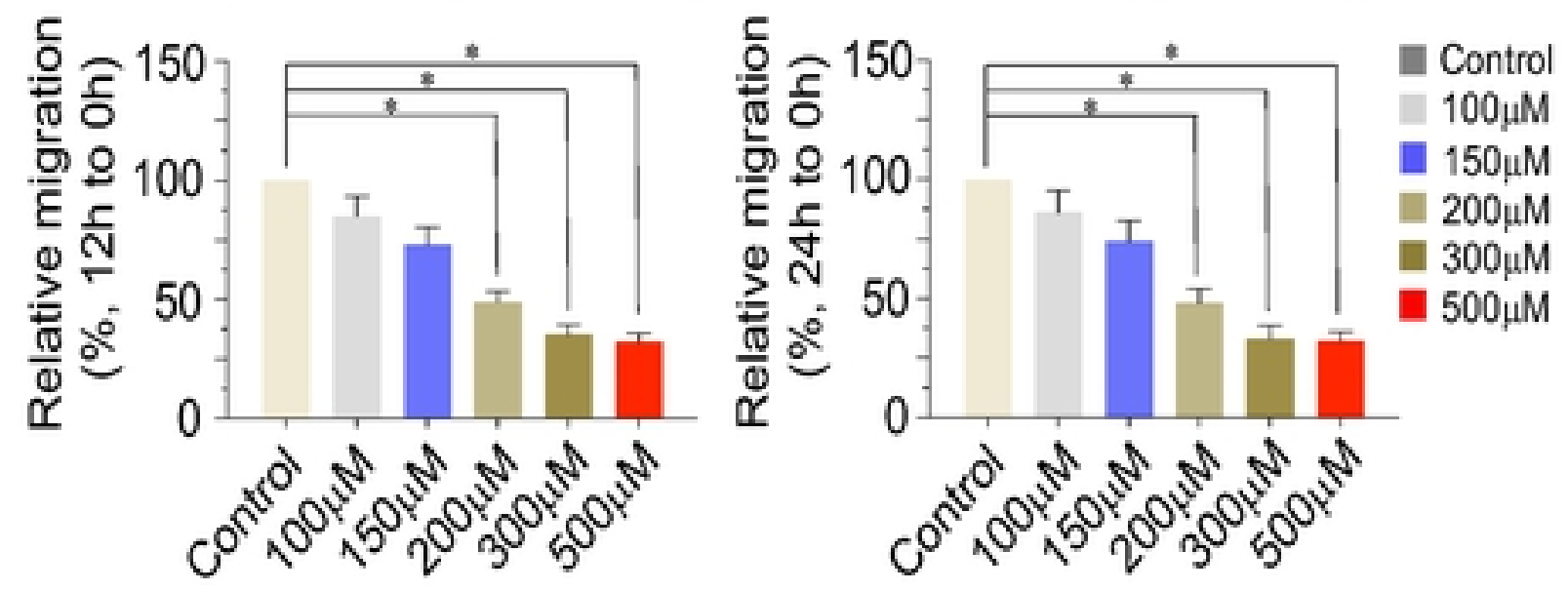

E

\section{MC3T3-E1 exposed to formaldehyde (FA) for $12 \mathrm{~h}$}

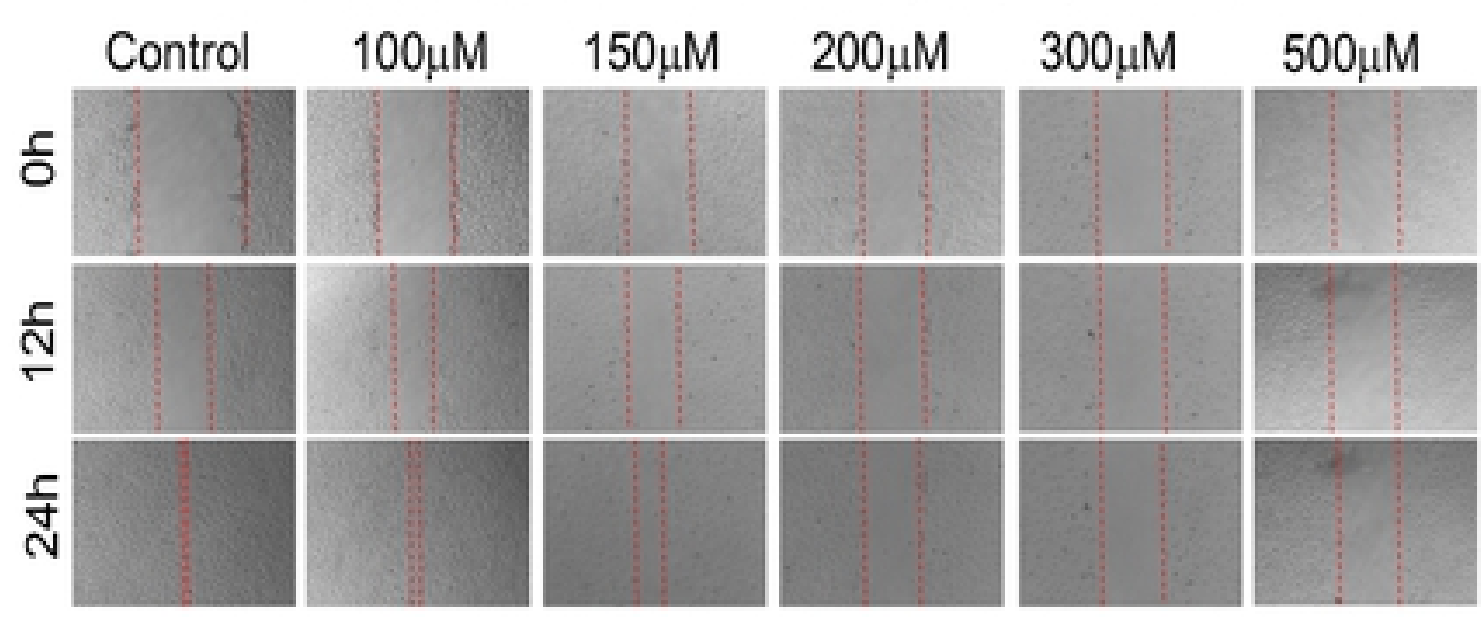

$\mathbf{F}$

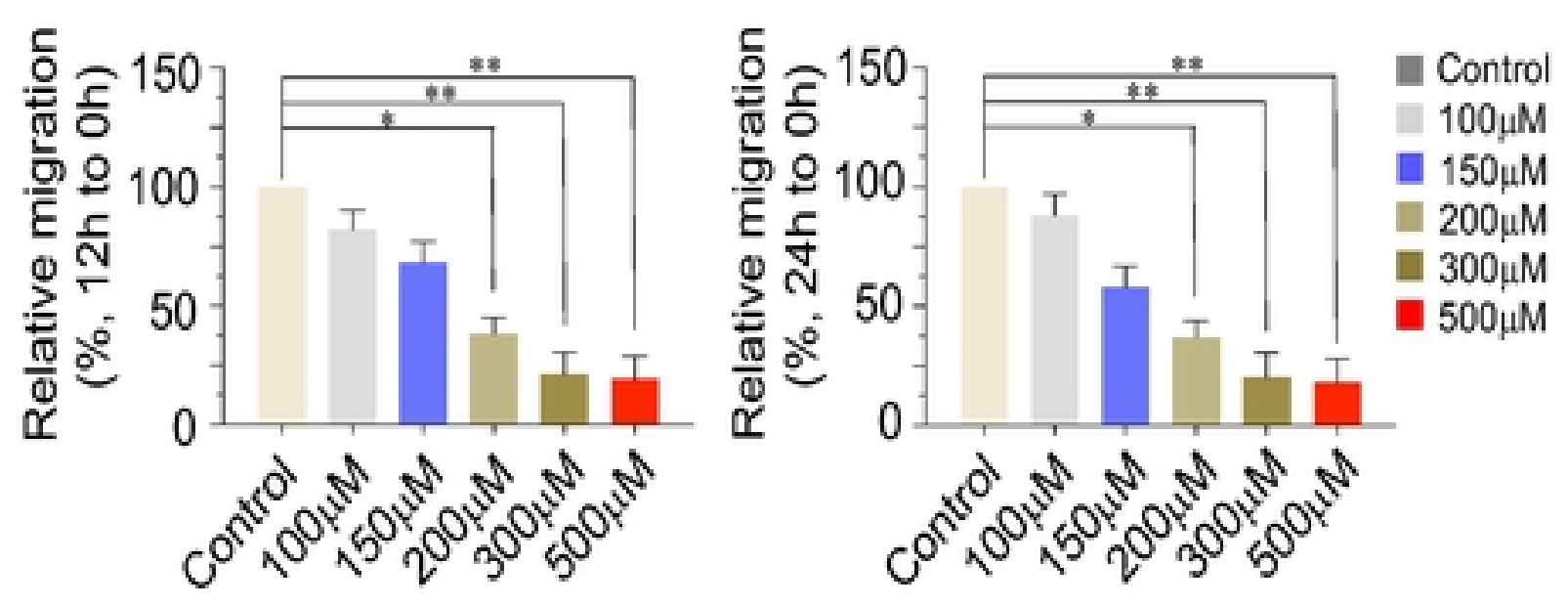

Figure-2 


\section{A}

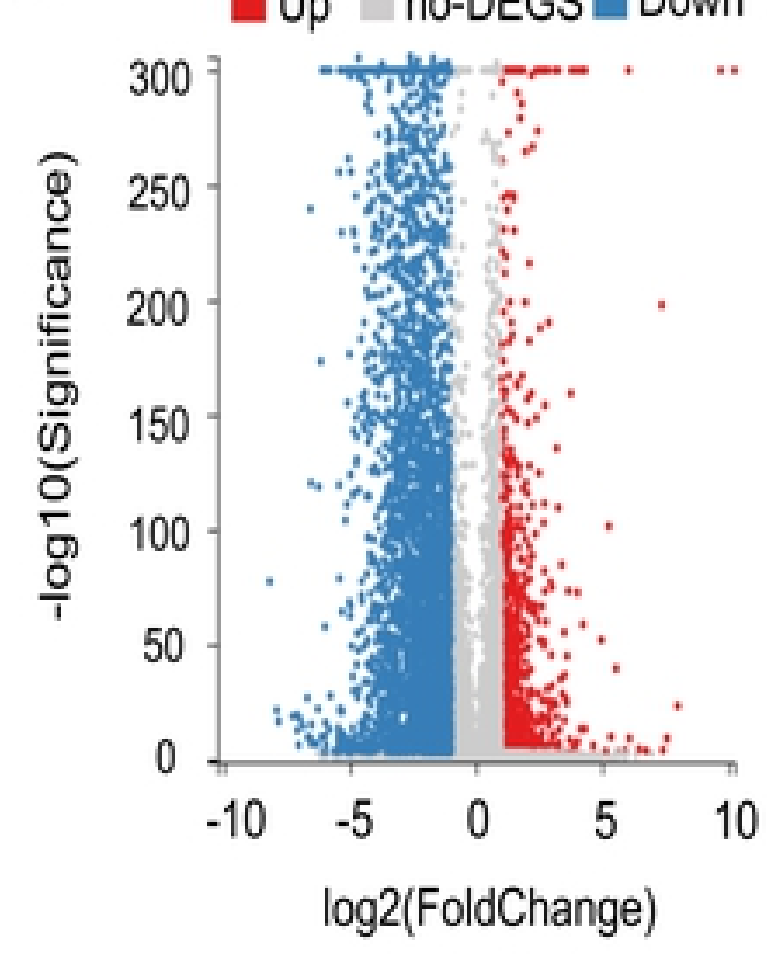

C

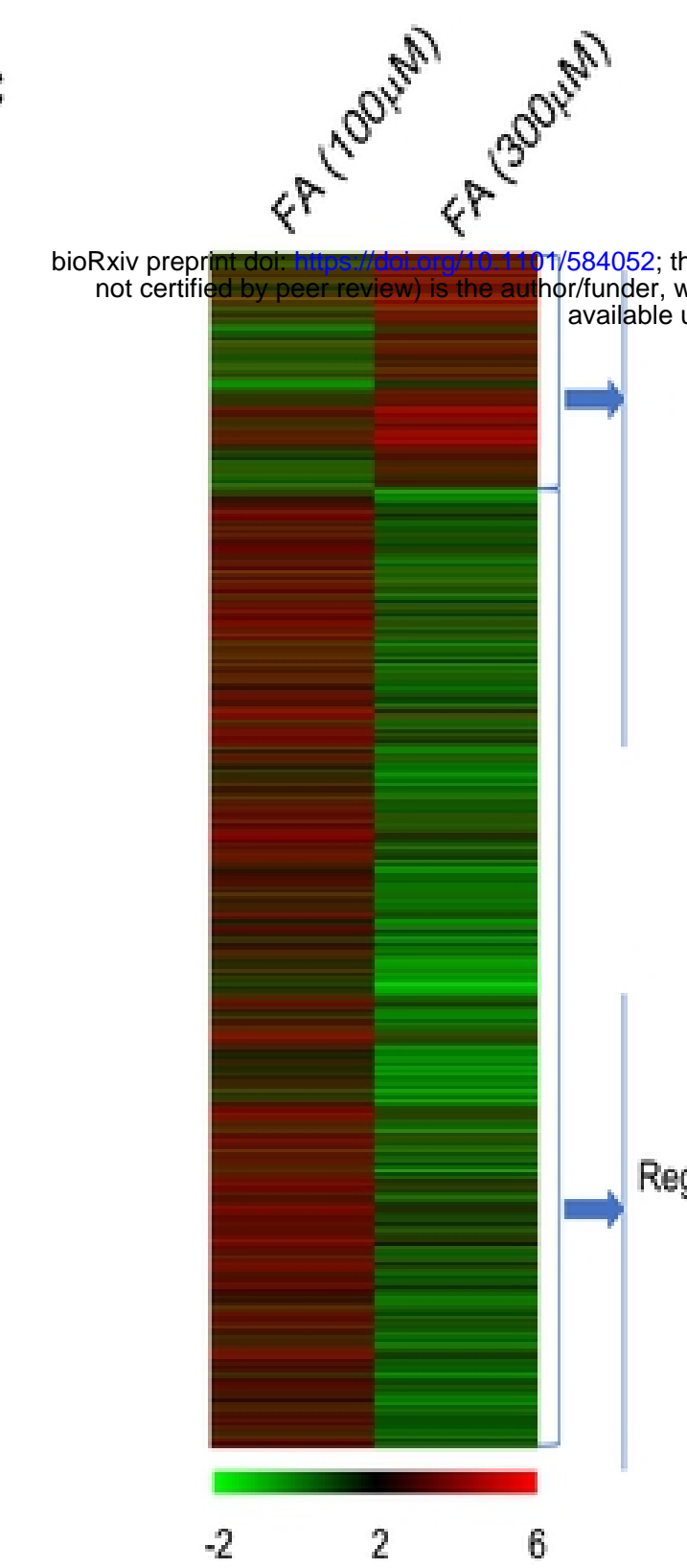

D
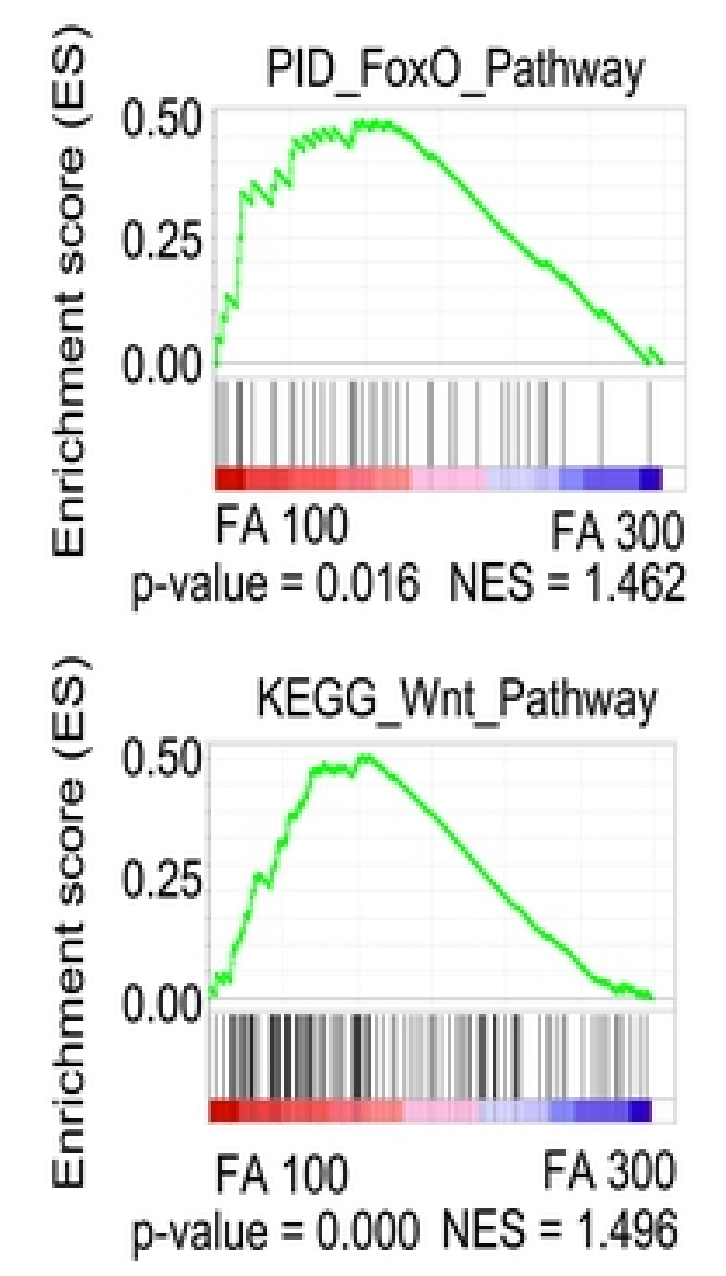

B

Adherens junction -

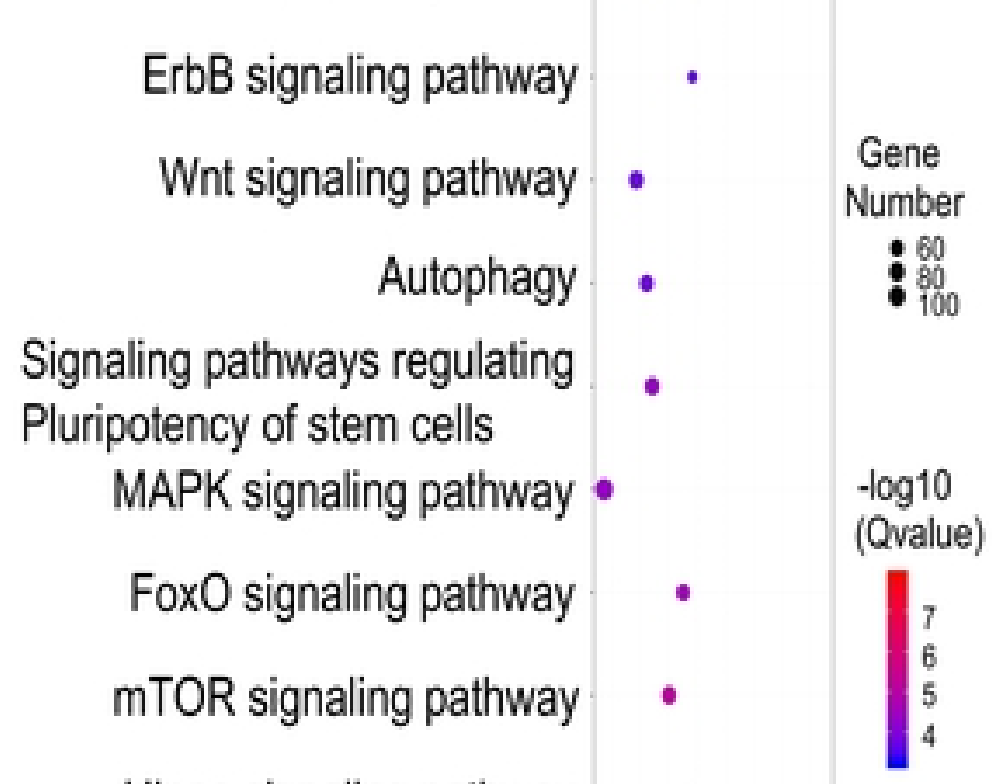

Hippo signaling pathway

p53 signaling pathway
Number

80

$\log 10$

egulating Pivipotency of

Hippo signaling pathway

mTOR signaling pathway

Fanconi anemia pathway

Biosynthesis of amino acids

Nucleotide excision repair

Glycolysis/Gluconeogenesis

Carbon metabolism

Term Candidate Gene Number

0.0 .0 .0 .60

Rich Ratio

$\begin{array}{llllll}0 & 5 & 10 & 15 & 20 & 25\end{array}$
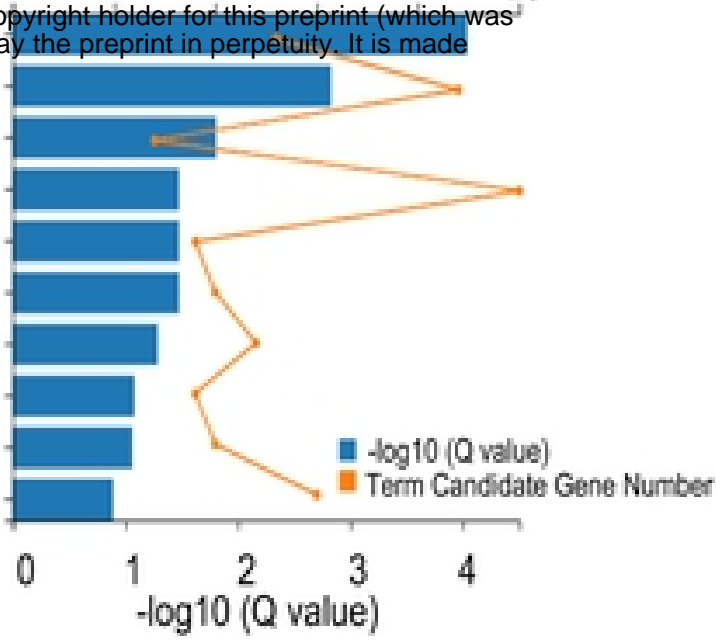

Term Candidate Gene Number

\begin{tabular}{lllll}
0 & 20 & 40 & 60 & 80 \\
\hline
\end{tabular}
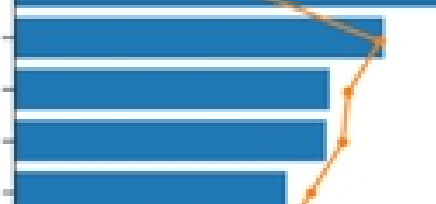

FoxO signaling pathway Autophagy MAPK signaling pathway

ErbB signaling pathway

Wnt signaling pathway TGF-beta signaling pathway
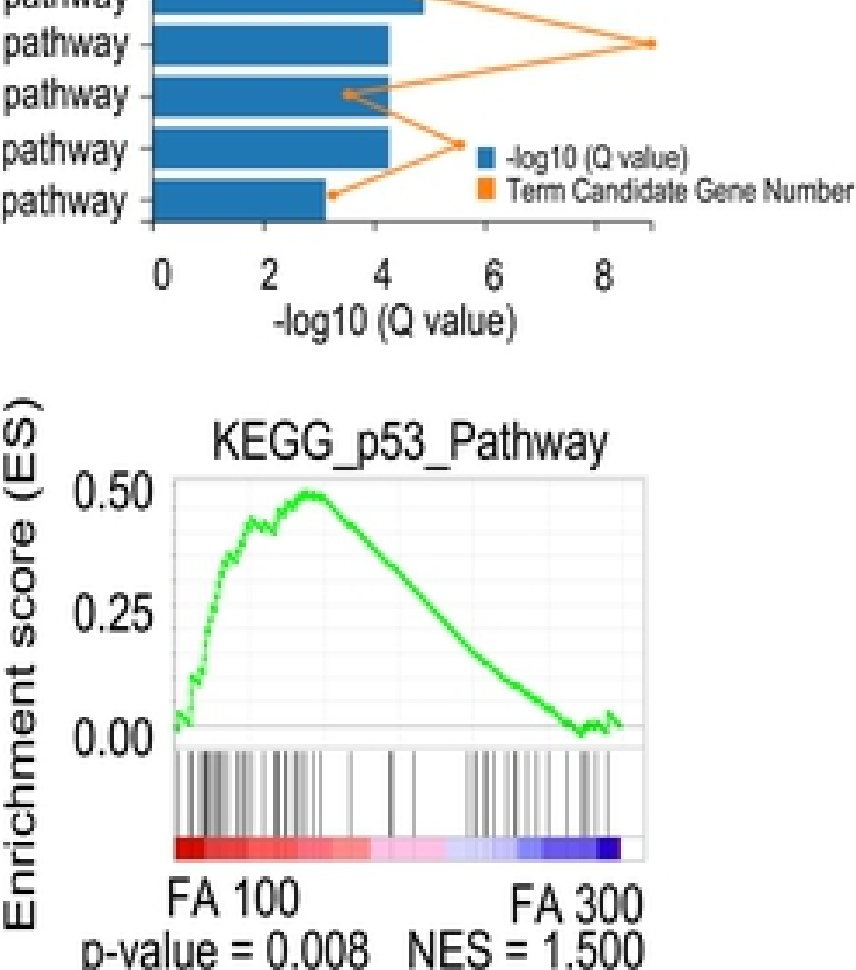

$\mathrm{p}$-value $=0.008 \quad \mathrm{NES}=1.500$

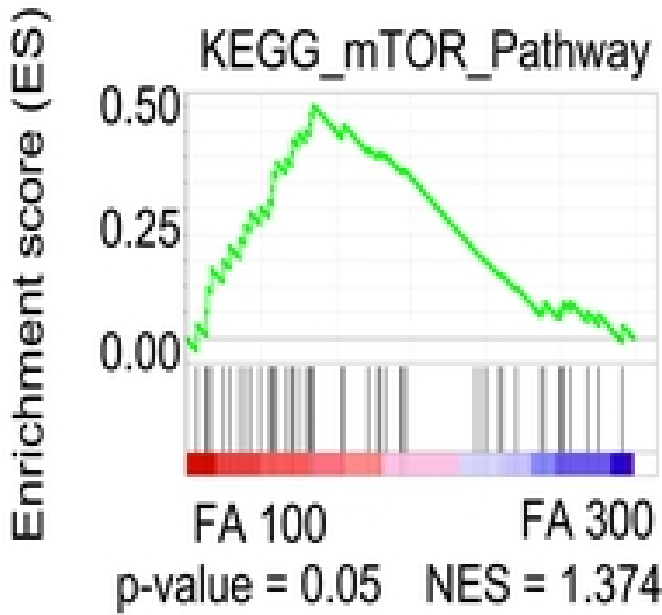

\section{Figure-3}


Fig. 4.

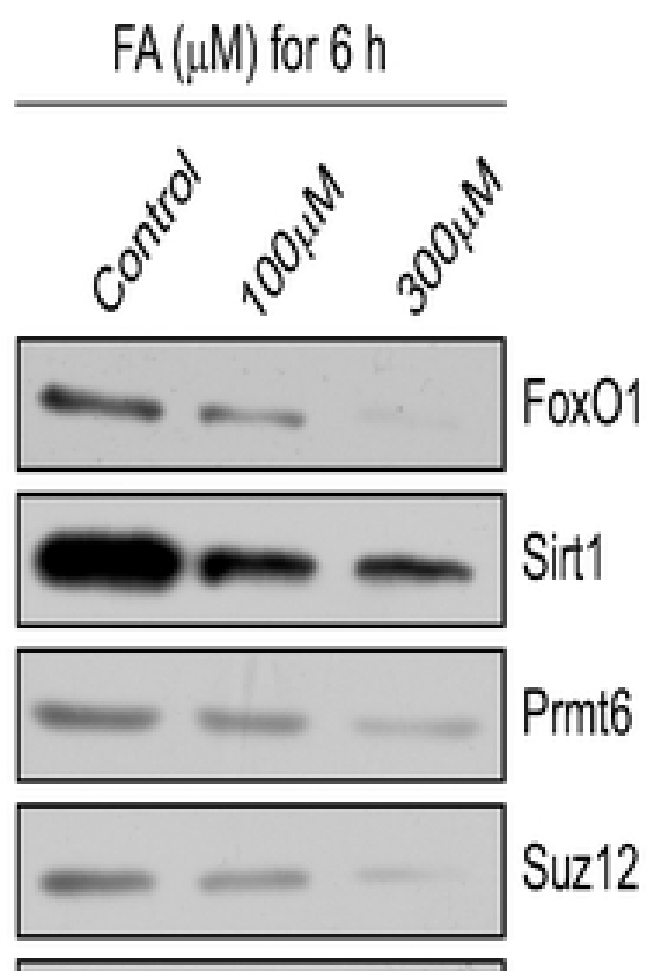

bioRxiv preprint doi: https://doi.org 10.1101/584052; this version posted Marefel29,2019. The copyright holder for this preprint (which was not certified by peer review) is the author/funder, who has granted bioRxiv a license to display the preprint in perpetuity. It is made available under aCC-BY 4.0 international license.

B-actin

Figure-4 